\title{
Addressing the COVID-19 Pandemic Among Persons Experiencing Homelessness: Steps to Protect a Vulnerable Population
}

\author{
Joshua A. Barocas, MD ${ }^{1,2}$, Karen R. Jacobson, MD, MPH ${ }^{1,2}$, and Davidson H. Hamer, \\ $M D^{1,2,3}$
}

\begin{abstract}
${ }^{1}$ Section of Infectious Diseases, Boston Medical Center (BMC), Boston, MA, USA; 2Department of Medicine, Boston University School of Medicine, Boston, MA, USA; ${ }^{3}$ Department of Global Health, Boston University School of Public Health, Boston, MA, USA.
\end{abstract}

J Gen Intern Med 36(5):1416-7

DOI: $10.1007 / \mathrm{s} 11606-020-06434-5$

(c) Society of General Internal Medicine 2021

$\mathrm{M}$ ore than half a million people in the USA experience homelessness nightly. ${ }^{1}$ Amidst the COVID-19 pandemic that has already widened existing health disparities among vulnerable populations across the country, this number could worsen with skyrocketing unemployment coupled with an end to the federal eviction moratorium. At least 1 in 5 of the 13.8 million adults in rental housing report being behind in rent, ${ }^{2}$ meaning a potential increase of more than 2.7 million newly homeless or unstably housed persons in the USA. Furthermore, medical conditions such as heart and lung disease that disproportionately affect persons experiencing homelessness also place them at risk for high morbidity and mortality from COVID-19. High infection transmission rates in shelters and a growing population experiencing homelessness result in increased COVID-19 risk across communities, not just for people experiencing homelessness. A sudden increase in the number of people without housing or infections in the existing homeless population combined with COVID-19's current strain on our health care system will greatly reduce our ability to care for this vulnerable population. There are at least four aspects of this crisis that need to be addressed to protect this vulnerable population and, simultaneously, the rest of the US population.

First, we need immediate improvements to and expansion of the national shelter system. Currently, it is incompatible with safety and unable to accommodate a growing homeless population. In shelters, where overcrowding is common, adhering to the recommended six-foot perimeter is impossible. Lack of personal protective equipment, such as face masks, for shelter guests and staff, and inadequate ventilation systems make transmission more likely. Furthermore, many

Received August 12, 2020

Accepted December 9, 2020

Published online February 2, 2021 individuals have no shelter options. The unhoused population in the USA, driven largely by unaffordable housing in urban areas, has grown substantially over the last 10 years. The situation will become more dire if the federal eviction moratorium is not extended. Federal and state relief plans must allocate funding for additional shelters that are properly staffed and resourced. Furthermore, the federal eviction moratorium needs to be extended with rent relief provided upon its expiration or we will suffer from the public health ramifications.

Second, we need to improve ongoing surveillance to prevent outbreaks among persons experiencing homelessness. To limit transmission, many shelters have instituted symptom screening protocols that include temperature screens and symptom recall for cough, sore throat, runny nose, and fatigue. These have proven to be largely ineffective as a sole means of prevention because persons with COVID-19 can remain asymptomatic or have a pre-symptomatic period of contagiousness. During a time of high community transmission, surveillance reports showed that as many as $66 \%$ of residents and staff at homeless shelters in three major US cities were positive for SARS-CoV- $2^{3}$ - orders of magnitude higher than general population estimates, ${ }^{3}$ the majority of which reported no symptoms. Municipalities should consider alternative surveillance techniques to monitor for outbreaks in real-time. One possible population-based surveillance technique utilizes municipal sewage systems. Waste water-based epidemiology is the practice of measuring biomarkers in wastewater and is a source of real-time geolocalized, unidentified personal data. ${ }^{4}$ Active surveillance of municipal wastewater mapped to homeless shelters could be used to identify insipient outbreaks. This is emerging as a potentially low-cost, feasible COVID-19 surveillance method that could be used in low-resource settings such as shelters.

Third, we need to develop a universal approach to testing and contact tracing. This gap is not unique to this population, but is one that has especially high barriers for successful implementation. For testing, lack of sufficient supplies has led to disproportionate allocation of tests across society, with more socially disadvantaged individuals encountering more challenges in accessing tests. Where universal testing is not available, random sampling at shelters on a scheduled basis or 
the pooling of samples could be used. For contact tracing, many new methods being deployed rely on the assumption of stable housing, secure internet, or cell phones with application capabilities. A majority of this population own or have access to a mobile phone, but most do not have smartphones that could support app-based programs and the overwhelming majority do not have an annual contract, meaning contact tracing programs may not be effective. ${ }^{5}$ We need a dedicated investment in contract tracing in this population; otherwise, expanded testing will be for naught.

Fourth, we need to provide places for persons with inadequate housing and without a permanent home to isolate once diagnosed with COVID-19 and not meeting criteria for skilled-medical care. Only one-quarter of the US population live in housing that is suitable for isolation and quarantine, including having sufficient bedrooms and bathrooms. ${ }^{6}$ Shelters are largely not equipped to convert entire floors into COVID-19 isolation or quarantine units. In Boston, we opened recuperation units and converted wards to accommodate this population and offload the skilled-medical system, but this required significant resource and personnel investments. ${ }^{7}$ There is a need for innovative approaches to help this vulnerable population attain a space to recuperate and limit spread, such as temporary lodging in hotels and university dormitories. Roadmaps and blueprints for these temporary locations exist, but there needs to be community-wide pressure to create the political will necessary for their implementation in places where outbreaks exist and readiness (e.g., financial resources, staffing protocols, space allocation) in places with outbreak potential.

As a nation, we have, thus far, done little to protect persons experiencing homelessness from COVID-19 disease. In the short term, we need funding for the expansion and improvement of our shelter systems, development and implementation of innovative strategies for active surveillance of outbreaks, rapid deployment of more COVID-19 tests coupled with a comprehensive contact tracing strategy, and expanded space for recuperation for this population. For a long-term effect, we need to extend the eviction moratoria and to use the pandemic as an opportunity to expand affordable and low-income housing and establish pathways to regain housing. Continued neglect of this vulnerable population will most certainly lead to considerable strain on the already stretched healthcare system during times of SARS-CoV-2 surge, increased transmission and mortality from SARS-CoV-2, and a widening health disparity gap.

Acknowledgments: We would like to thank Dr. Tara Bouton for her valuable comments and feedback on this piece.

Corresponding Author: Joshua A. Barocas, MD; Section of Infectious Diseases, Boston Medical Center (BMC), Boston, MA, USA (e-mail: Joshua.Barocas@BMC.org).

\section{Compliance with Ethical Standards:}

Conflict of Interest: The authors declare that they do not have a conflict of interest.

\section{REFERENCES}

1. National Alliance to End Homelessness. State of Homelessness. 2020. https://endhomelessness.org/homelessness-in-america/homelessnessstatistics/state-of-homelessness-report/. Accessed 1 Aug 2020.

2. US Bureau of Labor Statistics. Civilian unemployment rate. Secondary Civilian unemployment rate 2020. https://www.bls.gov/charts/employment-situation/civilian-unemployment-rate.htm. Accessed 31 July 2020.

3. Mosites E, Parker EM, Clarke KEN, et al. Assessment of SARS-CoV-2 infection prevalence in homeless shelters - four U.S. cities, March 27-April 15, 2020. MMWR Morb Mortal Wkly Rep. 2020;69(17):521-522.

4. Peccia J, Zulli A, Brackney DE, et al. SARS-CoV-2 RNA concentrations in primary municipal sewage sludge as a leading indicator of COVID-19 outbreak dynamics. medRxiv. 2020:2020.2005.2019.20105999.

5. Raven MC, Kaplan LM, Rosenberg M, Tieu L, Guzman D, Kushel M. Mobile phone, computer, and internet use among older homeless adults: results from the HOPE HOME cohort study. JMIR Mhealth Uhealth. 2018;6(12):e10049.

6. Sehgal AR, Himmelstein DU, Woolhandler S. Feasibility of separate rooms for home isolation and quarantine for COVID-19 in the United States. Ann Intern Med. 2020

7. Gaeta JM, De Las Nueces D, Munson DG, Barocas JA, Walsh KE. Case 21-2020: A 66-year-old homeless man with COVID-19. N Engl J Med. 2020;383(2):170-178.

Publisher's Note: Springer Nature remains neutral with regard to jurisdictional claims in published maps and institutional affiliations. 